\title{
Jupiter Analogs Orbit Stars with an Average Metallicity Close to That of the Sun
}

Buchhave, Lars A.; Bitsch, Bertram; Johansen, Anders; Latham, David W.; Bizzarro, Martin; Bieryla, Allyson; Kipping, David M.

Published in:

Astrophysical Journal

Link to article, DOI:

10.3847/1538-4357/aaafca

Publication date:

2018

Document Version

Publisher's PDF, also known as Version of record

Link back to DTU Orbit

Citation (APA):

Buchhave, L. A., Bitsch, B., Johansen, A., Latham, D. W., Bizzarro, M., Bieryla, A., \& Kipping, D. M. (2018). Jupiter Analogs Orbit Stars with an Average Metallicity Close to That of the Sun. Astrophysical Journal, 856(1), [37]. https://doi.org/10.3847/1538-4357/aaafca

\section{General rights}

Copyright and moral rights for the publications made accessible in the public portal are retained by the authors and/or other copyright owners and it is a condition of accessing publications that users recognise and abide by the legal requirements associated with these rights.

- Users may download and print one copy of any publication from the public portal for the purpose of private study or research.

- You may not further distribute the material or use it for any profit-making activity or commercial gain

- You may freely distribute the URL identifying the publication in the public portal 


\title{
Jupiter Analogs Orbit Stars with an Average Metallicity Close to That of the Sun
}

\author{
Lars A. Buchhave ${ }^{1}$ (1), Bertram Bitsch ${ }^{2}$ (1) , Anders Johansen $^{2}$, David W. Latham ${ }^{3}$ (1), \\ Martin Bizzarro $^{4}$, Allyson Bieryla ${ }^{3}$ (D), and David M. Kipping ${ }^{5}$ (D) \\ ${ }^{1}$ DTU Space, National Space Institute, Technical University of Denmark, Elektrovej 328, DK-2800 Kgs. Lyngby, Denmark; buchhave@space.dtu.dk \\ ${ }^{2}$ Lund Observatory, Lund University, Box 43, SE-221 00 Lund, Sweden \\ ${ }^{3}$ Harvard-Smithsonian Center for Astrophysics, 60 Garden Street, Cambridge, MA 02138, USA \\ ${ }^{4}$ Centre for Star and Planet Formation, Natural History Museum of Denmark, University of Copenhagen, ster Voldgade 5-7, DK-1350 Copenhagen K, Denmark \\ ${ }^{5}$ Department of Astronomy, Columbia University, 550 W. 120th Street, New York NY 10027, USA \\ Received 2017 December 27; revised 2018 February 12; accepted 2018 February 13; published 2018 March 22
}

\begin{abstract}
Jupiter played an important role in determining the structure and configuration of the Solar System. Whereas hotJupiter type exoplanets preferentially form around metal-rich stars, the conditions required for the formation of planets with masses, orbits, and eccentricities comparable to Jupiter (Jupiter analogs) are unknown. Using spectroscopic metallicities, we show that stars hosting Jupiter analogs have an average metallicity close to solar, in contrast to their hot-Jupiter and eccentric cool-Jupiter counterparts, which orbit stars with super-solar metallicities. Furthermore, the eccentricities of Jupiter analogs increase with host-star metallicity, suggesting that planet-planet scatterings producing highly eccentric cool Jupiters could be more common in metal-rich environments. To investigate a possible explanation for these metallicity trends, we compare the observations to numerical simulations, which indicate that metal-rich stars typically form multiple Jupiters, leading to planet-planet interactions and, hence, a prevalence of either eccentric cool Jupiters or hot Jupiters with circularized orbits. Although the samples are small and exhibit variations in their metallicities, suggesting that numerous processes other than metallicity affect the formation of planetary systems, the data in hand suggests that Jupiter analogs and terrestrial-sized planets form around stars with average metallicities close to solar, whereas high-metallicity systems preferentially host eccentric cool Jupiter or hot Jupiters, indicating that higher metallicity systems may not be favorable for the formation of planetary systems akin to the Solar System.
\end{abstract}

Key words: planetary systems - stars: abundances - surveys - techniques: spectroscopic

\section{Introduction}

Jupiter had a significant impact on the evolution of the Solar System shaping its structure and configuration (Tsiganis et al. 2005) and possibly reducing the accretion rate of terrestrial planets by limiting inward transport of solids (Kooten et al. 2016; Morbidelli et al. 2016). Furthermore, gravitational perturbations from Jupiter were likely responsible for seeding the Earth with water-rich asteroids (Morbidelli et al. 2012). Recent observational advances in the exoplanet field have revealed scores of closely packed multi-planet systems with short orbital periods and have shown that terrestrial-sized exoplanets are abundant (Borucki et al. 2010; Mayor et al. 2011). However, it is still unclear if the configuration of the Solar System with the inner region populated by terrestrial planets and the outer region populated by ice and gas giants, is common.

Gas giant planets with masses, orbits, and eccentricities analogous to Jupiter are difficult to detect due to their long orbital periods, but recent discoveries have increased the population of these Jupiter analogs enabling studies of the environment which they are formed. It is well-established that host-star metallicity, a proxy for the amount of heavy elements available to form planets in protoplanetary disks, is one of the driving factors determining the outcome of planet formation. The planet-metallicity correlation was first studied for hotJupiter type gas giant planets (Santos et al. 2004; Fischer \& Valenti 2005) and later for smaller sub-Neptune sized planets (Sousa et al. 2011; Buchhave et al. 2012, 2014). However, the gas giant studies were restricted to shorter period planets (e.g., periods $<4$ years; Fischer \& Valenti 2005) and consequently the conditions promoting the formation of planetary systems containing Jupiter analogs are poorly understood. This information is critical to constrain planet formation theories and place the Solar System in the context of a multitude of different types of planetary systems that exist in the Galaxy.

Here, we present a homogeneous analysis of host-star metallicities for systems hosting planets with masses, orbits, and eccentricities comparable to that of Jupiter, enabled by recent discoveries of these types of planets. Such data provide insights into the formation pathways of the largest and most massive planets that may significantly impact the architecture of their host planetary systems.

\section{Observations and Sample}

We define Jupiter analogs to be planets with approximately the mass of Jupiter $\left(0.3 M_{\mathrm{J}}<M_{p}<3.0 M_{\mathrm{J}}\right.$, with $M_{\mathrm{J}}$ indicating the mass of Jupiter), with low-orbital eccentricities $(e<0.25)$ and receiving less than a quarter of the insolation of the Earth,

$$
S_{\text {eff }}=\frac{T_{\text {eff }}}{5778 \mathrm{~K}}\left(\frac{R_{\star}}{R_{\odot}}\right)^{2}\left(\frac{a}{1 \mathrm{au}}\right)^{-2} \frac{1}{\sqrt{1-e^{2}}}<0.25 S_{\oplus},
$$

with $S_{\oplus}$ indicating units of Earth insolation. The low-orbital eccentricity suggests that the systems are less likely to have undergone a period of strong dynamical instability.

The insolation requirement would, in the Solar System translate to an object having a semimajor axis of $a>2 \mathrm{au}$, which is beyond the snow line after the protoplanetary disk has evolved for a few million years (Bitsch et al. 2015a). Planetary growth rates are believed to increase outside of the water ice 
line due to the additional solid material in this region (Pollack et al. 1996) and the larger sizes of icy pebbles relative to silicate pebbles in the interior of the ice line (Morbidelli et al. 2015).

We used data from exoplanet.org ${ }^{6}$ to identify known Jupiter analog type exoplanets according to these criteria. We identified 20 Jupiter analogs and an additional 17 cool eccentric Jupiters with the same criteria except the requirement that the cool eccentric Jupiter have an orbital eccentricity higher than 0.25 .

We collected a total of 1889 high-resolution spectra, including both new observations and publicly available archival spectra, from four different spectrographs of the host stars of 35 systems containing the 37 Jupiter analogs and eccentric cool Jupiters as well as a representative comparison sample of similar size of hot-Jupiter host stars. The hot-Jupiter sample consists of 28 hot-Jupiter host stars from the HATNet transit survey and from hot-Jupiter planets identified by the Kepler Mission that already have published metallicities derived using the stellar parameter classification tool (SPC; Buchhave et al. 2014). The hot Jupiters were selected using the same mass restrictions as the Jupiter analogs and the additional requirement of a small semimajor axis $(a<0.1 \mathrm{au})$.

The spectra originate from the following four spectrographs: the fiber-fed Tillinghast Reflector Echelle Spectrograph (TRES; Fúrész 2008) on the $1.5 \mathrm{~m}$ Tillinghast Reflector at the Fred Lawrence Whipple Observatory on Mt. Hopkins, Arizona; the High Accuracy Radial velocity Planet Searcher (HARPS; Mayor et al. 2003) at the ESO La Silla $3.6 \mathrm{~m}$ telescope; the High Resolution Echelle Spectrograph (HIRES; Vogt et al. 1994) on the $10 \mathrm{~m}$ Keck I telescope at MaunaKea, Hawaii; and the FIber-fed chelle Spectrograph (FIES; Telting et al. 2014) on the $2.5 \mathrm{~m}$ Nordic Optical Telescope on La Palma, Spain. We gathered 21 new and 142 archival observations from TRES, 1689 public archival spectra from HARPS, 30 public archival spectra from HIRES, and 7 archival spectra from FIES, totaling 1889 spectra in all.

In order to minimize known biases associated with using different techniques to derive host-star metallicities, we analyzed the gathered spectra of the host stars using the SPC tool (Buchhave et al. 2012, 2014), deriving spectroscopic metallicities in a homogeneous and consistent manner for the entire sample of stars. SPC has been utilized in a large number of exoplanet publications and has been compared with other classification techniques by a number of independent research groups (e.g., Huber et al. 2014; Wang et al. 2016; Petigura et al. 2017).

We removed a total of four of the Jupiter analogs due to the reasons described in the following. SPC provides reliable stellar parameters for solar-type stars, but it has not been rigorously tested for evolved stars and cooler dwarf stars. We therefore restricted our analysis to solar-like dwarf stars $\left(T_{\text {eff }}>4500 \mathrm{~K}\right.$ and $\left.\log g>4.0\right)$ and thus removed two stars (HD 114613 and HD 11964) due to their low surface gravity $(\log g<4.0)$. Second, we were unable to obtain appropriate spectra of the two stars HD 154857 and HIP 57274 during the observing time available to us. The sample of hot Jupiters also adheres to these restrictions on effective temperature and surface gravity.

\footnotetext{
From exoplanets.org on 2016 June 17.
}

We have compared our metallicities of the Jupiter analogs and the hot Jupiters to the published values on exoplanet.org and we find an average difference of -0.01 dex and an rms of the difference between our metallicities and the published metallicities of $0.07 \mathrm{dex}$, consistent with the formal uncertainties. Furthermore, we compared our metallicities to the SWEET-Cat database of stellar parameters ${ }^{7}$ (Santos et al. 2013) and found similar good agreement with an average difference of -0.03 dex and an rms of the difference between our metallicities and the SWEET-Cat metallicities of $0.07 \mathrm{dex}$. Table 1 contains the stellar parameters derived using SPC for all of the planet-hosting stars used in this article as well as values used in this manuscript from exoplanet.org at the time we downloaded them (2016 June 17). Furthermore, we used previously published SPC metallicities for the Kepler hotJupiter planets (Buchhave et al. 2014).

To understand the formation history of the systems, including whether these have undergone dynamical planetplanet interactions that would result in large eccentricities, the planets are categorized using the eccentricity of the discovered planet with the maximum eccentricity in the system. However, when using the eccentricities of the individual planets, we find very similar results.

\section{Spectroscopic Metallicities}

In Figure 1 we show that the average host-star metallicity of Jupiter analogs is close to $\operatorname{solar}^{8}([\mathrm{~m} / \mathrm{H}]=-0.07 \pm$ $0.05( \pm 0.21)$ ), where the uncertainty is the standard error of the mean and the standard deviation is shown in parentheses, whereas the host stars of the hot Jupiters and eccentric cool Jupiters are, on average, metal-rich $([\mathrm{m} / \mathrm{H}]=+0.25 \pm 0.03$ $( \pm 0.16)$ and $[\mathrm{m} / \mathrm{H}]=+0.23 \pm 0.04( \pm 0.14)$, respectively). The average metallicity of the hot-Jupiter hosts is similar to the average metallicity of hot-Jupiter hosts from the radial velocity (RV) studies and the Kepler Mission analyzed in previous studies (RV hot Jupiters $[\mathrm{m} / \mathrm{H}]=+0.23 \pm 0.03$, Fischer \& Valenti 2005; and Kepler gas giants $[\mathrm{m} / \mathrm{H}]=+0.18 \pm 0.02$, Buchhave et al. 2014). A two-sample Kolmogorov-Smirnov $(\mathrm{K}-\mathrm{S})$ test reveals that the null hypothesis that the sample of stars orbited by Jupiter analogs and the sample of stars orbited by hot Jupiters are drawn from the same parent population and can be rejected with $99.996 \%$ confidence $(4.1 \sigma, p$-value $=$ $4.26 \times 10^{-5}$ ). Using the eccentricity of the individual planets as opposed to the maximum eccentricity in each system, we find that the confidence level of the $\mathrm{K}-\mathrm{S}$ test is slightly lower at $3.9 \sigma$. We also calculated the two independent sample t-test to determine the probability that two-sample populations have significantly different means. For the Jupiter analogs and hot Jupiters, we find that the null hypothesis can be rejected with 99.999\% confidence $\left(4.3 \sigma, p\right.$-value $\left.=1.44 \times 10^{-5}\right)$ and with $99.997 \%$ confidence $\left(4.2 \sigma, p\right.$-value $\left.=2.65 \times 10^{-5}\right)$ when using the eccentricity of the individual planets.

Figure 2 shows the eccentricities of the Jupiter analogs plotted against their host-star metallicities. The cool Jupiters with larger eccentricities are found orbiting host stars that are more metal-rich, whereas Jupiter analogs with orbital eccentricities closer to circular in systems with only one discovered

\footnotetext{
7 https://www.astro.up.pt/resources/sweet-cat

8 SPC utilizes all of the absorption lines in the wavelength region 505 to $536 \mathrm{~nm}$ and we therefore denote the metallicities in this work as $[\mathrm{m} / \mathrm{H}]$, representing a mix of metals assumed to be the same as the relative pattern of the abundances in the Sun.
} 
Table 1

Parameters for Jupiter Analogs, Eccentric Cool Jupiters, and Hot Jupiters

\begin{tabular}{|c|c|c|c|c|c|c|c|c|c|c|c|}
\hline Star & $\mathrm{pl}$ & Teff & {$[\mathrm{m} / \mathrm{H}]$} & $\log g$ & ncomp & ecc & Max ecc & $m \sin i$ & Period & $a$ & $R_{\star}$ \\
\hline \multicolumn{12}{|c|}{ Jupiter analogs } \\
\hline 47UMa & $\mathrm{c}$ & $5825 \pm 50$ & $-0.06 \pm 0.08$ & $4.26 \pm 0.10$ & 2 & $0.098 \pm 0.071$ & 0.098 & $0.546 \pm 0.071$ & $2391.0 \pm 93.5$ & $3.572 \pm 0.111$ & $1.119 \pm 0.038$ \\
\hline HD 114386 & $\mathrm{~b}$ & $4928 \pm 50$ & $0.00 \pm 0.08$ & $4.62 \pm 0.10$ & 1 & $0.230 \pm 0.030$ & 0.230 & $1.364 \pm 0.079$ & $937.7 \pm 15.6$ & $1.727 \pm 0.035$ & $0.637 \pm 0.029$ \\
\hline HD 117207 & $\mathrm{~b}$ & $5644 \pm 50$ & $0.18 \pm 0.08$ & $4.27 \pm 0.10$ & 1 & $0.144 \pm 0.035$ & 0.144 & $1.819 \pm 0.089$ & $2597.0 \pm 41.0$ & $3.738 \pm 0.074$ & $0.952 \pm 0.034$ \\
\hline HD 12661 & c & $5776 \pm 50$ & $0.38 \pm 0.08$ & $4.42 \pm 0.10$ & 2 & $0.031 \pm 0.022$ & 0.377 & $1.949 \pm 0.092$ & $1707.9 \pm 13.9$ & $2.919 \pm 0.064$ & $1.068 \pm 0.037$ \\
\hline HD 134987 & $\mathrm{c}$ & $5715 \pm 50$ & $0.27 \pm 0.08$ & $4.23 \pm 0.10$ & 2 & $0.120 \pm 0.020$ & 0.233 & $0.805 \pm 0.046$ & $5000.0 \pm 400.0$ & $5.825 \pm 0.331$ & $1.166 \pm 0.039$ \\
\hline HD 13931 & $\mathrm{~b}$ & $5789 \pm 50$ & $-0.06 \pm 0.08$ & $4.21 \pm 0.10$ & 1 & $0.020 \pm 0.035$ & 0.020 & $1.881 \pm 0.142$ & $4218.0 \pm 388.0$ & $5.149 \pm 0.327$ & $1.224 \pm 0.042$ \\
\hline HD 154345 & $\mathrm{~b}$ & $5559 \pm 50$ & $-0.14 \pm 0.08$ & $4.59 \pm 0.10$ & 1 & $0.044 \pm 0.045$ & 0.044 & $0.957 \pm 0.061$ & $3341.6 \pm 92.7$ & $4.214 \pm 0.105$ & $1.000 \pm 0.050$ \\
\hline HD 164922 & $\mathrm{~b}$ & $5424 \pm 50$ & $0.11 \pm 0.08$ & $4.48 \pm 0.10$ & 1 & $0.050 \pm 0.095$ & 0.050 & $0.358 \pm 0.060$ & $1155.0 \pm 23.0$ & $2.101 \pm 0.045$ & $0.899 \pm 0.033$ \\
\hline HD 222155 & $\mathrm{~b}$ & $5763 \pm 50$ & $-0.13 \pm 0.08$ & $4.10 \pm 0.10$ & 1 & $0.160 \pm 0.215$ & 0.160 & $2.026 \pm 0.500$ & $3999.0 \pm 505.0$ & $5.139 \pm 0.464$ & $1.670 \pm 0.070$ \\
\hline HD 25171 & $\mathrm{~b}$ & $6063 \pm 50$ & $-0.18 \pm 0.08$ & $4.17 \pm 0.10$ & 1 & $0.080 \pm 0.060$ & 0.080 & $0.956 \pm 0.234$ & $1845.0 \pm 167.0$ & $3.031 \pm 0.190$ & $1.069 \pm 0.041$ \\
\hline HD 290327 & $\mathrm{~b}$ & $5571 \pm 50$ & $-0.11 \pm 0.08$ & $4.42 \pm 0.10$ & 1 & $0.080 \pm 0.055$ & 0.080 & $2.548 \pm 0.206$ & $2443.0 \pm 161.0$ & $3.431 \pm 0.161$ & $0.997 \pm 0.033$ \\
\hline HD 37124 & c & $5510 \pm 50$ & $-0.45 \pm 0.08$ & $4.35 \pm 0.10$ & 3 & $0.125 \pm 0.055$ & 0.160 & $0.648 \pm 0.055$ & $885.5 \pm 5.1$ & $1.710 \pm 0.029$ & $0.772 \pm 0.030$ \\
\hline HD 37124 & d & $5510 \pm 50$ & $-0.45 \pm 0.08$ & $4.35 \pm 0.10$ & 3 & $0.160 \pm 0.140$ & 0.160 & $0.687 \pm 0.075$ & $1862.0 \pm 38.0$ & $2.807 \pm 0.060$ & $0.772 \pm 0.030$ \\
\hline HD 4208 & $\mathrm{~b}$ & $5556 \pm 50$ & $-0.37 \pm 0.08$ & $4.24 \pm 0.10$ & 1 & $0.052 \pm 0.040$ & 0.052 & $0.807 \pm 0.041$ & $828.0 \pm 8.1$ & $1.654 \pm 0.030$ & $0.877 \pm 0.032$ \\
\hline HD 47186 & $\mathrm{c}$ & $5650 \pm 50$ & $0.19 \pm 0.08$ & $4.29 \pm 0.10$ & 2 & $0.249 \pm 0.073$ & 0.249 & $0.348 \pm 0.076$ & $1353.6 \pm 57.1$ & $2.387 \pm 0.078$ & $1.131 \pm 0.037$ \\
\hline HD 6718 & $\mathrm{~b}$ & $5726 \pm 50$ & $-0.11 \pm 0.08$ & $4.37 \pm 0.10$ & 1 & $0.100 \pm 0.075$ & 0.100 & $1.559 \pm 0.117$ & $2496.0 \pm 176.0$ & $3.554 \pm 0.177$ & $0.957 \pm 0.031$ \\
\hline HD 70642 & $\mathrm{~b}$ & $5635 \pm 50$ & $0.12 \pm 0.08$ & $4.27 \pm 0.10$ & 1 & $0.034 \pm 0.039$ & 0.034 & $1.909 \pm 0.104$ & $2068.0 \pm 39.0$ & $3.181 \pm 0.066$ & $1.031 \pm 0.036$ \\
\hline HD 8535 & $\mathrm{~b}$ & $6042 \pm 50$ & $-0.06 \pm 0.08$ & $4.18 \pm 0.10$ & 1 & $0.150 \pm 0.070$ & 0.150 & $0.682 \pm 0.052$ & $1313.0 \pm 28.0$ & $2.445 \pm 0.054$ & $1.044 \pm 0.036$ \\
\hline HD 89307 & $\mathrm{~b}$ & $5925 \pm 50$ & $-0.19 \pm 0.08$ & $4.33 \pm 0.10$ & 1 & $0.200 \pm 0.050$ & 0.200 & $1.791 \pm 0.150$ & $2166.0 \pm 38.0$ & $3.266 \pm 0.067$ & $1.151 \pm 0.039$ \\
\hline epsilonEri & $\mathrm{b}$ & $5026 \pm 50$ & $-0.19 \pm 0.08$ & $4.39 \pm 0.10$ & 1 & $0.250 \pm 0.230$ & 0.250 & $1.054 \pm 0.188$ & $2500.0 \pm 350.0$ & $3.376 \pm 0.322$ & $0.740 \pm 0.010$ \\
\hline muAra & $\mathrm{c}$ & $5727 \pm 50$ & $0.26 \pm 0.08$ & $4.20 \pm 0.10$ & 4 & $0.099 \pm 0.063$ & 0.172 & $1.889 \pm 0.223$ & $4205.8 \pm 458.9$ & $5.341 \pm 0.402$ & $1.250 \pm 0.042$ \\
\hline \multicolumn{12}{|c|}{ Jupiter analogs with expanded mass limits } \\
\hline $55 \mathrm{Cnc}$ & $\mathrm{d}$ & $5382 \pm 50$ & $0.38 \pm 0.08$ & $4.44 \pm 0.10$ & 5 & $0.020 \pm 0.008$ & 0.320 & $3.545 \pm 0.122$ & $4909.0 \pm 30.0$ & $5.475 \pm 0.094$ & $0.943 \pm 0.010$ \\
\hline HD 10180 & $\mathrm{~h}$ & $5806 \pm 50$ & $-0.01 \pm 0.08$ & $4.23 \pm 0.10$ & 6 & $0.151 \pm 0.072$ & 0.151 & $0.206 \pm 0.016$ & $2248.0 \pm 104.0$ & $3.425 \pm 0.120$ & $1.109 \pm 0.036$ \\
\hline HD 111232 & $\mathrm{~b}$ & $5521 \pm 50$ & $-0.36 \pm 0.08$ & $4.44 \pm 0.10$ & 1 & $0.200 \pm 0.010$ & 0.200 & $6.842 \pm 0.251$ & $1143.0 \pm 14.0$ & $1.975 \pm 0.037$ & $0.875 \pm 0.042$ \\
\hline HD 128311 & c & $5033 \pm 50$ & $0.05 \pm 0.08$ & $4.66 \pm 0.10$ & 2 & $0.230 \pm 0.058$ & 0.345 & $3.248 \pm 0.159$ & $923.8 \pm 5.3$ & $1.745 \pm 0.030$ & $0.583 \pm 0.028$ \\
\hline HD 183263 & $\mathrm{c}$ & $5853 \pm 50$ & $0.20 \pm 0.08$ & $4.25 \pm 0.10$ & 2 & $0.239 \pm 0.064$ & 0.357 & $3.476 \pm 0.309$ & $3066.0 \pm 110.0$ & $4.295 \pm 0.125$ & $1.117 \pm 0.038$ \\
\hline HD 204313 & $\mathrm{~b}$ & $5677 \pm 50$ & $0.11 \pm 0.08$ & $4.22 \pm 0.10$ & 2 & $0.230 \pm 0.040$ & 0.280 & $3.501 \pm 0.221$ & $1920.1 \pm 25.0$ & $3.071 \pm 0.058$ & $1.126 \pm 0.038$ \\
\hline HD 24040 & $\mathrm{~b}$ & $5741 \pm 50$ & $0.09 \pm 0.08$ & $4.20 \pm 0.10$ & 1 & $0.040 \pm 0.065$ & 0.040 & $4.022 \pm 0.326$ & $3668.0 \pm 170.0$ & $4.924 \pm 0.206$ & $1.154 \pm 0.039$ \\
\hline HD 30177 & $\mathrm{~b}$ & $5642 \pm 50$ & $0.42 \pm 0.08$ & $4.32 \pm 0.10$ & 1 & $0.193 \pm 0.025$ & 0.193 & $9.688 \pm 0.544$ & $2770.0 \pm 100.0$ & $3.808 \pm 0.134$ & $1.212 \pm 0.041$ \\
\hline HD 37605 & c & $5477 \pm 50$ & $0.31 \pm 0.08$ & $4.50 \pm 0.10$ & 2 & $0.013 \pm 0.014$ & 0.677 & $3.366 \pm 1.124$ & $2720.0 \pm 57.0$ & $3.818 \pm 0.638$ & $0.917 \pm 0.030$ \\
\hline HD 72659 & $\mathrm{~b}$ & $5893 \pm 50$ & $-0.07 \pm 0.08$ & $4.16 \pm 0.10$ & 1 & $0.220 \pm 0.030$ & 0.220 & $3.174 \pm 0.148$ & $3658.0 \pm 32.0$ & $4.754 \pm 0.084$ & $1.343 \pm 0.044$ \\
\hline HD 95872 & $\mathrm{~b}$ & $5361 \pm 50$ & $0.31 \pm 0.08$ & $4.56 \pm 0.10$ & 1 & $0.060 \pm 0.040$ & 0.060 & $4.594 \pm 0.352$ & $4375.0 \pm 169.0$ & $5.154 \pm 0.158$ & $0.999 \pm 0.048$ \\
\hline \multicolumn{12}{|c|}{ Eccentric cool Jupiters } \\
\hline HD 108874 & $\mathrm{c}$ & $5577 \pm 50$ & $0.15 \pm 0.08$ & $4.32 \pm 0.10$ & 2 & $0.273 \pm 0.040$ & 0.273 & $1.028 \pm 0.054$ & $1680.4 \pm 23.9$ & $2.720 \pm 0.052$ & $1.121 \pm 0.038$ \\
\hline HD 126614A & $\mathrm{b}$ & $5598 \pm 50$ & $0.50 \pm 0.08$ & $4.28 \pm 0.10$ & 1 & $0.410 \pm 0.100$ & 0.410 & $0.386 \pm 0.044$ & $1244.0 \pm 17.0$ & $2.368 \pm 0.045$ & $1.110 \pm 0.039$ \\
\hline HD 128311 & $\mathrm{~b}$ & $5033 \pm 50$ & $0.05 \pm 0.08$ & $4.66 \pm 0.10$ & 2 & $0.345 \pm 0.049$ & 0.345 & $1.457 \pm 0.152$ & $454.2 \pm 1.6$ & $1.086 \pm 0.018$ & $0.583 \pm 0.028$ \\
\hline HD 171238 & $\mathrm{~b}$ & $5557 \pm 50$ & $0.18 \pm 0.08$ & $4.55 \pm 0.10$ & 1 & $0.400 \pm 0.060$ & 0.400 & $2.609 \pm 0.148$ & $1523.0 \pm 43.0$ & $2.543 \pm 0.064$ & $1.051 \pm 0.046$ \\
\hline HD 181433 & $\mathrm{c}$ & $4914 \pm 50$ & $0.36 \pm 0.08$ & $4.35 \pm 0.10$ & 3 & $0.280 \pm 0.020$ & 0.480 & $0.640 \pm 0.027$ & $962.0 \pm 15.0$ & $1.756 \pm 0.034$ & $1.008 \pm 0.070$ \\
\hline HD 181433 & d & $4914 \pm 50$ & $0.36 \pm 0.08$ & $4.35 \pm 0.10$ & 3 & $0.480 \pm 0.050$ & 0.480 & $0.535 \pm 0.051$ & $2172.0 \pm 158.0$ & $3.022 \pm 0.155$ & $1.008 \pm 0.070$ \\
\hline HD 190360 & $\mathrm{~b}$ & $5572 \pm 50$ & $0.21 \pm 0.08$ & $4.26 \pm 0.10$ & 2 & $0.313 \pm 0.019$ & 0.313 & $1.535 \pm 0.061$ & $2915.0 \pm 28.9$ & $3.973 \pm 0.071$ & $1.075 \pm 0.037$ \\
\hline HD 202206 & c & $5740 \pm 50$ & $0.24 \pm 0.08$ & $4.43 \pm 0.10$ & 2 & $0.267 \pm 0.021$ & 0.435 & $2.331 \pm 0.127$ & $1383.4 \pm 18.4$ & $2.490 \pm 0.055$ & $0.986 \pm 0.035$ \\
\hline HD 204313 & $\mathrm{~d}$ & $5677 \pm 50$ & $0.11 \pm 0.08$ & $4.22 \pm 0.10$ & 2 & $0.280 \pm 0.090$ & 0.280 & $1.606 \pm 0.281$ & $2831.6 \pm 150.0$ & $3.945 \pm 0.154$ & $0.000 \pm 0.000$ \\
\hline HD 207832 & c & $5712 \pm 50$ & $0.10 \pm 0.08$ & $4.44 \pm 0.10$ & 2 & $0.270 \pm 0.160$ & 0.270 & $0.730 \pm 0.161$ & $1155.7 \pm 54.5$ & $2.112 \pm 0.100$ & $0.901 \pm 0.056$ \\
\hline HD 217107 & c & $5732 \pm 50$ & $0.37 \pm 0.08$ & $4.44 \pm 0.10$ & 2 & $0.517 \pm 0.033$ & 0.517 & $2.615 \pm 0.150$ & $4270.0 \pm 220.0$ & $5.334 \pm 0.204$ & $1.500 \pm 0.030$ \\
\hline HD 220773 & $\mathrm{~b}$ & $5878 \pm 50$ & $-0.01 \pm 0.08$ & $4.10 \pm 0.10$ & 1 & $0.510 \pm 0.100$ & 0.510 & $1.450 \pm 0.251$ & $3724.7 \pm 463.0$ & $4.943 \pm 0.418$ & $1.351 \pm 0.049$ \\
\hline HD 50499 & $\mathrm{~b}$ & $6008 \pm 50$ & $0.29 \pm 0.08$ & $4.29 \pm 0.10$ & 1 & $0.254 \pm 0.203$ & 0.254 & $1.745 \pm 0.140$ & $2457.9 \pm 37.9$ & $3.872 \pm 0.076$ & $1.184 \pm 0.040$ \\
\hline
\end{tabular}


Table 1

(Continued)

\begin{tabular}{|c|c|c|c|c|c|c|c|c|c|c|c|}
\hline Star & $\mathrm{pl}$ & Teff & {$[\mathrm{m} / \mathrm{H}]$} & $\log g$ & ncomp & ecc & Max ecc & $m \sin i$ & Period & $a$ & $R_{\star}$ \\
\hline HD 66428 & $\mathrm{~b}$ & $5749 \pm 50$ & $0.31 \pm 0.08$ & $4.41 \pm 0.10$ & 1 & $0.465 \pm 0.030$ & 0.465 & $2.750 \pm 0.195$ & $1973.0 \pm 31.0$ & $3.143 \pm 0.070$ & $0.980 \pm 0.034$ \\
\hline HD 79498 & $\mathrm{~b}$ & $5786 \pm 50$ & $0.25 \pm 0.08$ & $4.46 \pm 0.10$ & 1 & $0.590 \pm 0.020$ & 0.590 & $1.346 \pm 0.073$ & $1966.1 \pm 41.0$ & $3.133 \pm 0.068$ & $1.128 \pm 0.044$ \\
\hline HD 87883 & $\mathrm{~b}$ & $5043 \pm 50$ & $0.02 \pm 0.08$ & $4.61 \pm 0.10$ & 1 & $0.530 \pm 0.120$ & 0.530 & $1.756 \pm 0.282$ & $2754.0 \pm 87.0$ & $3.576 \pm 0.096$ & $0.778 \pm 0.038$ \\
\hline \multicolumn{12}{|c|}{ Eccentric cool Jupiters with expanded mass limits } \\
\hline HD 106252 & $\mathrm{~b}$ & $5870 \pm 50$ & $-0.12 \pm 0.08$ & $4.34 \pm 0.10$ & 1 & $0.482 \pm 0.011$ & 0.482 & $6.959 \pm 0.257$ & $1531.0 \pm 4.7$ & $2.611 \pm 0.044$ & $1.123 \pm 0.038$ \\
\hline HD 108341 & $\mathrm{~b}$ & $5225 \pm 50$ & $0.16 \pm 0.08$ & $4.59 \pm 0.10$ & 1 & $0.850 \pm 0.085$ & 0.850 & $3.477 \pm 4.643$ & $1129.0 \pm 7.0$ & $2.007 \pm 0.034$ & $0.790 \pm 0.030$ \\
\hline HD 204941 & $\mathrm{~b}$ & $5126 \pm 50$ & $-0.13 \pm 0.08$ & $4.58 \pm 0.10$ & 1 & $0.370 \pm 0.080$ & 0.370 & $0.267 \pm 0.035$ & $1733.0 \pm 74.0$ & $2.554 \pm 0.084$ & $0.850 \pm 0.037$ \\
\hline HD 73267 & $\mathrm{~b}$ & $5399 \pm 50$ & $0.10 \pm 0.08$ & $4.36 \pm 0.10$ & 1 & $0.256 \pm 0.009$ & 0.256 & $3.063 \pm 0.105$ & $1260.0 \pm 7.0$ & $2.198 \pm 0.038$ & $1.166 \pm 0.037$ \\
\hline HD 74156 & $\mathrm{c}$ & $5973 \pm 50$ & $0.00 \pm 0.08$ & $4.17 \pm 0.10$ & 2 & $0.380 \pm 0.020$ & 0.630 & $8.247 \pm 0.357$ & $2520.0 \pm 15.0$ & $3.900 \pm 0.067$ & $1.345 \pm 0.044$ \\
\hline \multicolumn{12}{|l|}{ Hot-Jupiters } \\
\hline HAT-P-1 & $\mathrm{b}$ & $5895 \pm 50$ & $0.04 \pm 0.08$ & $4.26 \pm 0.10$ & $\ldots$ & $\ldots$ & $\ldots$ & $\ldots$ & $\cdots$ & $\cdots$ & $\ldots$ \\
\hline HAT-P-13 & $\mathrm{b}$ & $5837 \pm 50$ & $0.50 \pm 0.08$ & $4.29 \pm 0.10$ & $\cdots$ & $\cdots$ & $\ldots$ & $\ldots$ & $\ldots$ & $\cdots$ & $\cdots$ \\
\hline HAT-P-15 & $\mathrm{b}$ & $5674 \pm 50$ & $0.29 \pm 0.08$ & $4.45 \pm 0.10$ & $\ldots$ & $\cdots$ & $\ldots$ & $\ldots$ & $\ldots$ & $\ldots$ & $\ldots$ \\
\hline HAT-P-16 & $\mathrm{b}$ & $6121 \pm 50$ & $0.05 \pm 0.08$ & $4.31 \pm 0.10$ & $\cdots$ & $\cdots$ & $\ldots$ & $\cdots$ & $\cdots$ & $\cdots$ & $\cdots$ \\
\hline HAT-P-17 & $\mathrm{b}$ & $5377 \pm 50$ & $0.09 \pm 0.08$ & $4.58 \pm 0.10$ & $\cdots$ & $\ldots$ & $\ldots$ & $\ldots$ & $\ldots$ & $\ldots$ & $\ldots$ \\
\hline НАТ-Р-19 & $\mathrm{b}$ & $5017 \pm 50$ & $0.34 \pm 0.08$ & $4.51 \pm 0.10$ & $\ldots$ & $\ldots$ & $\ldots$ & $\ldots$ & $\ldots$ & $\ldots$ & $\ldots$ \\
\hline HAT-P-20 & $\mathrm{b}$ & $4653 \pm 50$ & $0.22 \pm 0.08$ & $4.61 \pm 0.10$ & $\cdots$ & $\cdots$ & $\cdots$ & $\cdots$ & $\cdots$ & $\cdots$ & $\cdots$ \\
\hline HAT-P-21 & $\mathrm{b}$ & $5632 \pm 50$ & $0.04 \pm 0.08$ & $4.30 \pm 0.10$ & $\cdots$ & $\cdots$ & $\cdots$ & $\cdots$ & $\cdots$ & $\cdots$ & $\cdots$ \\
\hline HAT-P-22 & $\mathrm{b}$ & $5355 \pm 50$ & $0.26 \pm 0.08$ & $4.39 \pm 0.10$ & $\ldots$ & $\ldots$ & $\ldots$ & $\ldots$ & $\ldots$ & $\ldots$ & $\ldots$ \\
\hline HAT-P-23 & $\mathrm{b}$ & $5900 \pm 50$ & $0.09 \pm 0.08$ & $4.45 \pm 0.10$ & $\ldots$ & $\ldots$ & $\ldots$ & $\ldots$ & $\ldots$ & $\ldots$ & $\ldots$ \\
\hline HAT-P-25 & $\mathrm{b}$ & $5604 \pm 51$ & $0.43 \pm 0.08$ & $4.48 \pm 0.10$ & $\ldots$ & $\ldots$ & $\ldots$ & $\ldots$ & $\ldots$ & $\ldots$ & $\ldots$ \\
\hline HAT-P-27 & $\mathrm{b}$ & $5346 \pm 50$ & $0.31 \pm 0.08$ & $4.52 \pm 0.10$ & $\ldots$ & $\ldots$ & $\ldots$ & $\ldots$ & $\ldots$ & $\ldots$ & $\ldots$ \\
\hline HAT-P-29 & $\mathrm{b}$ & $6061 \pm 50$ & $0.11 \pm 0.08$ & $4.27 \pm 0.10$ & $\ldots$ & $\ldots$ & $\cdots$ & $\ldots$ & $\cdots$ & $\cdots$ & $\ldots$ \\
\hline HAT-P-3 & $\mathrm{b}$ & $5209 \pm 50$ & $0.35 \pm 0.08$ & $4.54 \pm 0.10$ & $\cdots$ & $\cdots$ & $\ldots$ & $\cdots$ & $\cdots$ & $\cdots$ & $\cdots$ \\
\hline HAT-P-4 & $\mathrm{b}$ & $6070 \pm 50$ & $0.33 \pm 0.08$ & $4.31 \pm 0.10$ & $\cdots$ & $\cdots$ & $\ldots$ & $\cdots$ & $\ldots$ & $\ldots$ & $\ldots$ \\
\hline HAT-P-44 & $\mathrm{b}$ & $5372 \pm 50$ & $0.37 \pm 0.08$ & $4.43 \pm 0.10$ & $\cdots$ & $\ldots$ & $\ldots$ & $\cdots$ & $\ldots$ & $\ldots$ & $\ldots$ \\
\hline HAT-P-46 & $\mathrm{b}$ & $6197 \pm 50$ & $0.31 \pm 0.08$ & $4.30 \pm 0.10$ & $\ldots$ & $\cdots$ & $\ldots$ & $\ldots$ & $\cdots$ & $\cdots$ & $\ldots$ \\
\hline K00001 & 01 & $5870 \pm 50$ & $0.05 \pm 0.08$ & $4.47 \pm 0.10$ & $\cdots$ & $\ldots$ & $\ldots$ & $\ldots$ & $\ldots$ & $\ldots$ & $\ldots$ \\
\hline K00017 & 01 & $5775 \pm 50$ & $0.48 \pm 0.08$ & $4.41 \pm 0.10$ & $\ldots$ & $\ldots$ & $\cdots$ & $\ldots$ & $\ldots$ & $\ldots$ & $\ldots$ \\
\hline K00022 & 01 & $5850 \pm 50$ & $0.16 \pm 0.08$ & $4.29 \pm 0.10$ & $\ldots$ & $\ldots$ & $\ldots$ & $\ldots$ & $\ldots$ & $\ldots$ & $\ldots$ \\
\hline K00094 & 01 & $6176 \pm 50$ & $0.01 \pm 0.08$ & $4.23 \pm 0.10$ & $\ldots$ & $\ldots$ & $\ldots$ & $\ldots$ & $\ldots$ & $\ldots$ & $\ldots$ \\
\hline K00098 & 01 & $6482 \pm 50$ & $0.01 \pm 0.08$ & $4.14 \pm 0.10$ & $\cdots$ & $\cdots$ & $\ldots$ & $\cdots$ & $\ldots$ & $\cdots$ & $\ldots$ \\
\hline K00127 & 01 & $5611 \pm 50$ & $0.34 \pm 0.08$ & $4.50 \pm 0.10$ & $\ldots$ & $\ldots$ & $\ldots$ & $\ldots$ & $\ldots$ & $\ldots$ & $\ldots$ \\
\hline K00128 & 01 & $5679 \pm 50$ & $0.34 \pm 0.08$ & $4.32 \pm 0.10$ & $\ldots$ & $\ldots$ & $\ldots$ & $\ldots$ & $\ldots$ & $\ldots$ & $\ldots$ \\
\hline K00135 & 01 & $6012 \pm 50$ & $0.35 \pm 0.08$ & $4.37 \pm 0.10$ & $\cdots$ & $\cdots$ & $\cdots$ & $\cdots$ & $\cdots$ & $\cdots$ & $\cdots$ \\
\hline K00203 & 01 & $5331 \pm 50$ & $0.50 \pm 0.08$ & $4.28 \pm 0.12$ & $\cdots$ & $\cdots$ & $\cdots$ & $\cdots$ & $\cdots$ & $\cdots$ & $\cdots$ \\
\hline K00351 & 01 & $5976 \pm 50$ & $0.11 \pm 0.08$ & $4.32 \pm 0.10$ & $\ldots$ & $\ldots$ & $\ldots$ & $\ldots$ & $\ldots$ & $\ldots$ & $\ldots$ \\
\hline K00433 & 02 & $5233 \pm 50$ & $0.29 \pm 0.08$ & $4.54 \pm 0.10$ & $\cdots$ & $\ldots$ & $\ldots$ & $\cdots$ & $\ldots$ & $\ldots$ & $\ldots$ \\
\hline K00682 & 01 & $5566 \pm 50$ & $0.34 \pm 0.08$ & $4.27 \pm 0.10$ & $\ldots$ & $\ldots$ & $\ldots$ & $\ldots$ & $\ldots$ & $\cdots$ & $\ldots$ \\
\hline K00686 & 01 & $5593 \pm 50$ & $0.06 \pm 0.09$ & $4.42 \pm 0.15$ & $\ldots$ & $\ldots$ & $\ldots$ & $\ldots$ & $\ldots$ & & $\ldots$ \\
\hline K01411 & 01 & $5718 \pm 50$ & $0.41 \pm 0.08$ & $4.35 \pm 0.10$ & $\ldots$ & $\ldots$ & $\ldots$ & $\ldots$ & $\ldots$ & $\ldots$ & $\ldots$ \\
\hline K03681 & 01 & $6258 \pm 50$ & $0.16 \pm 0.08$ & $4.39 \pm 0.10$ & $\ldots$ & $\ldots$ & $\ldots$ & $\ldots$ & $\ldots$ & $\ldots$ & $\ldots$ \\
\hline
\end{tabular}

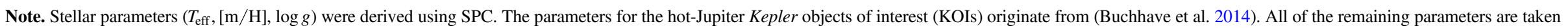
from exoplanets.org (2016 June 17). 

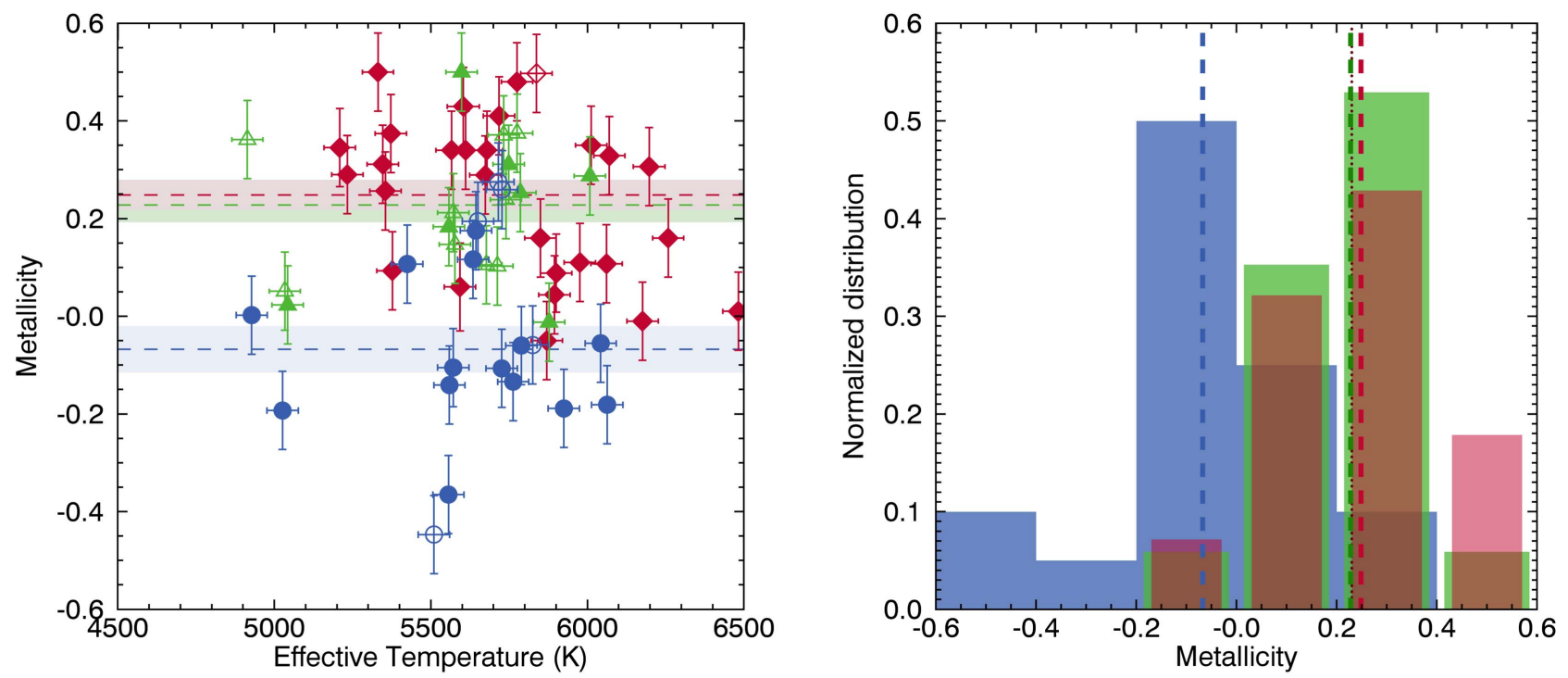

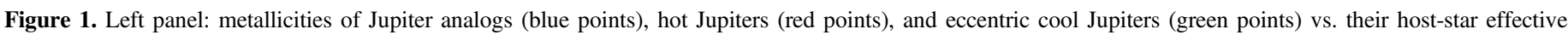

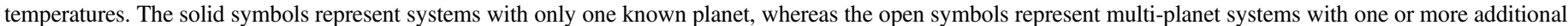

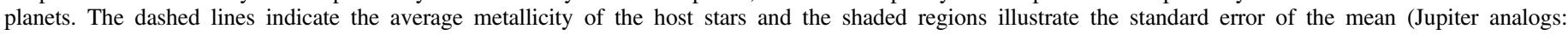

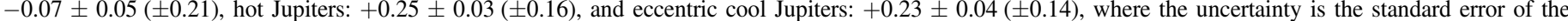

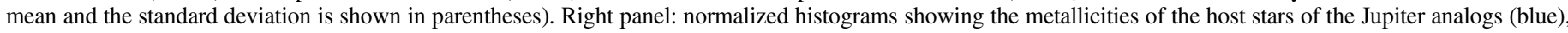

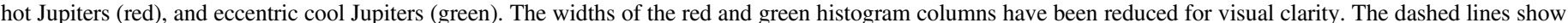

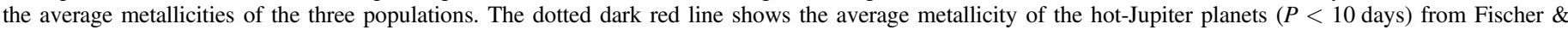

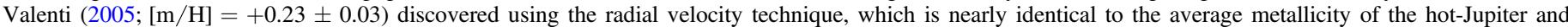

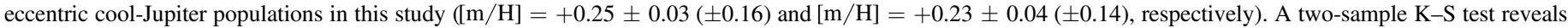

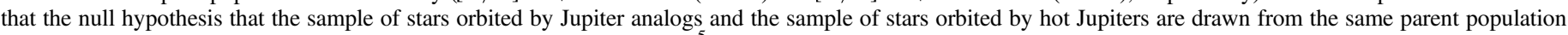

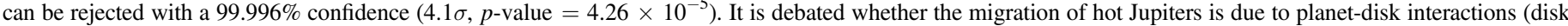

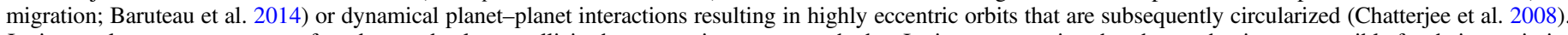

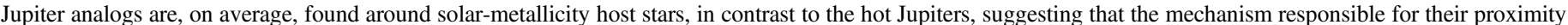
to the host stars must be more effective in high-metallicity environments.

planet are predominantly found orbiting host stars with metallicities closer to that of the Sun. When including systems with multiple planets and assigning the eccentricities of the individual planets (right panel, all points), the planets appear to form around a wider range of metallicities.

The eccentricities of Jupiter analogs thus depend on host-star metallicity and hence the initial metallicity of the protoplanetary disk, in agreement with previous findings for warm Jupiters (orbital distances of 0.1-1 au; Dawson \& Murray-Clay 2013) and for short-period planet candidates from Kepler (Shabram et al. 2016), suggesting that planet-planet scatterings producing highly eccentric cool Jupiters are more common in metal-rich environments. A two-sample $\mathrm{K}-\mathrm{S}$ test shows that the null hypothesis that the sample of stars orbited by Jupiter analogs and the sample of stars orbited by cool eccentric Jupiters are drawn from the same parent population can be rejected with $99.96 \%$ confidence $(3.6 \sigma, p$-value $=3.73 \times$ $10^{-4}$ ). Using the eccentricities of the individual planets rather than the maximum eccentricity of the system, the confidence level is $99.91 \%\left(3.3 \sigma, p\right.$-value $\left.=9.04 \times 10^{-4}\right)$. The t-test null hypothesis can be rejected with $99.994 \%$ confidence $(4.0 \sigma$, $p$-value $\left.=6.47 \times 10^{-5}\right)$ and with $99.987 \%$ confidence $(3.8 \sigma$, $p$-value $=1.29 \times 10^{-4}$ ) when using the eccentricity of the individual planets. To test the robustness of our results to larger and smaller gas giant masses, we expanded the mass range to $0.2 M_{\mathrm{J}}<M_{p}<13 M_{\mathrm{J}}$, which lead to similar results as above (see Section 5 for details). Furthermore, bootstrap with replacement simulations indicate that the results are robust to the removal of individual data points (see Section 5 for details).
Our results indicate that Jupiter analogs are found on average around stars with solar metallicity in contrast to the hot Jupiters that predominantly orbit metal-rich stars. Thus, the widely accepted hot-Jupiter metallicity correlation does not seem to extend to longer period gas giants with low eccentricity. Hot-Jupiter exoplanets are thought to form beyond the ice line and subsequently migrate inwards close to their host stars. However, it is debated whether their migration is due to planet-disk interactions (disk migration; Baruteau et al. 2014) or dynamical planet-planet interactions resulting in highly eccentric orbits that are subsequently circularized (Chatterjee et al. 2008). The results in Figure 1 suggest that the mechanism responsible for migration is more effective in high-metallicity regimes relative to environments with solar-like metallicities. Furthermore, Figure 2 shows that the eccentricity of Jupiter analogs is correlated with host-star metallicity and thus the initial metallicity of the protoplanetary disk, suggesting that planet-planet scatterings producing highly eccentric Jupiter analogs are more common in metalrich environments.

\section{Planet Formation Interpretation}

These findings provide insights into the formation mechanisms responsible for gas giant planet formation and, as such, we performed numerical simulations of planet formation by pebble accretion to interpret our observations. We emphasize that this is one of many possible interpretations and should be viewed as a model that is consistent with the observed metallicity trends. Recent work has shown that rapid formation of planetary cores 

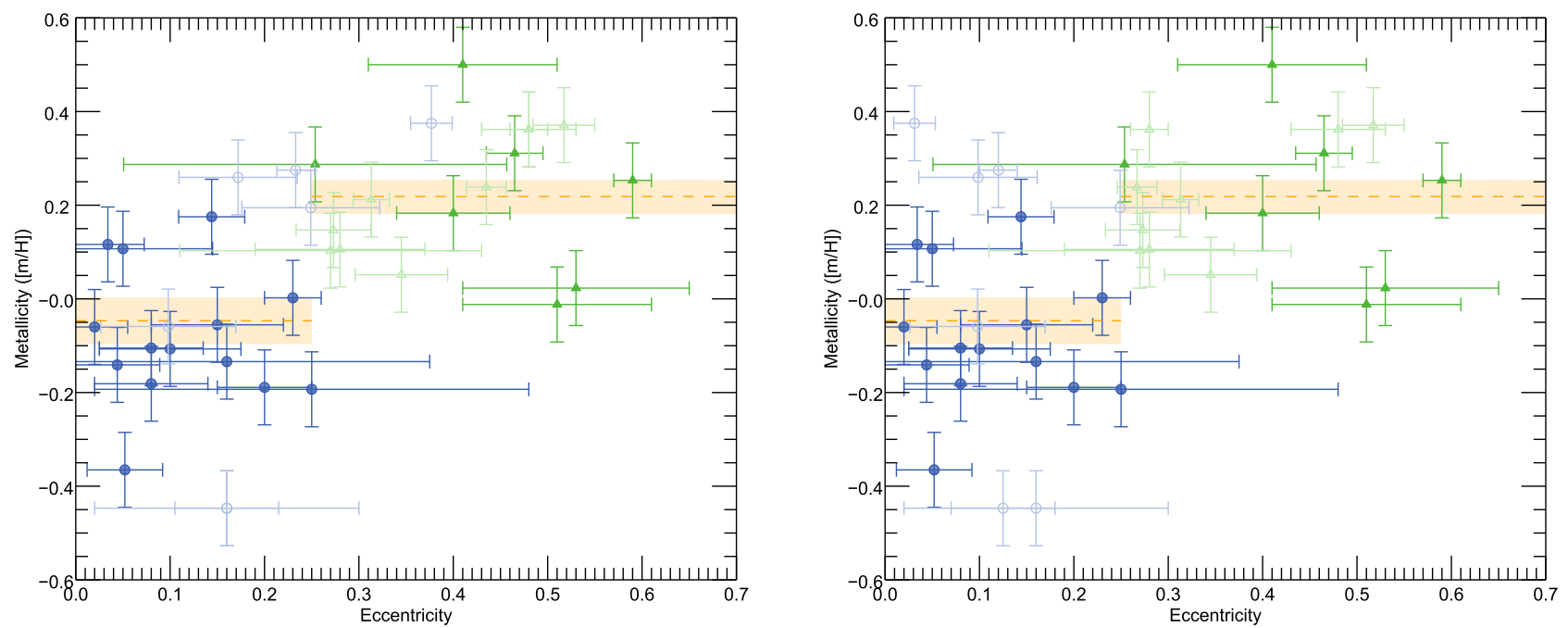

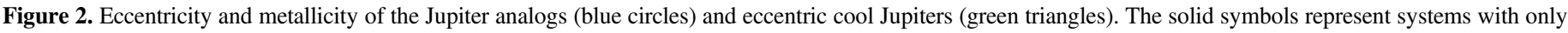

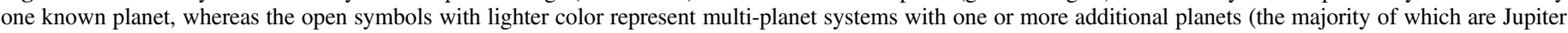

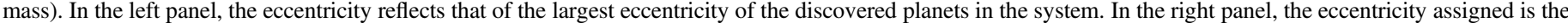

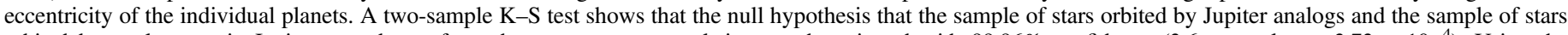

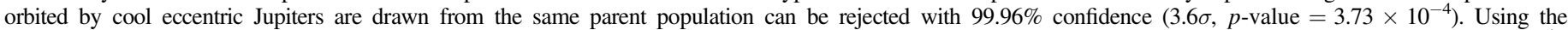

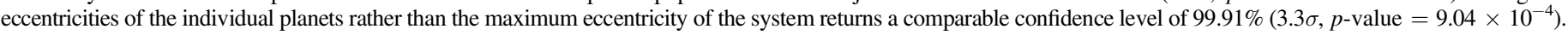

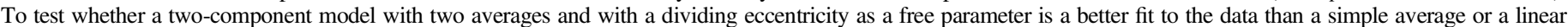

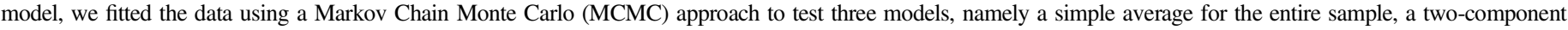

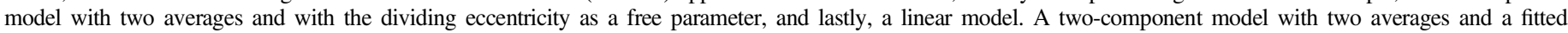

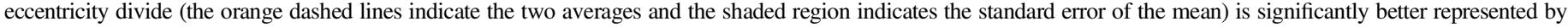
the data than a model with a simple average $(\triangle \mathrm{BIC}=120$, see Section 5 for details).

within the lifetime of protoplanetary disks can occur by pebble accretion, that is, the accretion of centimeter- to meter-sized particles loosely bound to the gas onto planetesimals seeds (Ormel \& Klahr 2010; Lambrechts \& Johansen 2012; Lambrechts et al. 2014; Bitsch et al. 2015b; Levison et al. 2015). Astronomical observations indicate that pebbles are abundant in the protoplanetary disks of dust and gas orbiting young stars (Brauer et al. 2007). Moreover, pebble accretion allows for the efficient formation of planetary cores in disks with a wide range of metallicity as well as out to large orbital distances (10-20 au; Lambrechts \& Johansen 2014; Levison et al. 2015). We note that our results could also be understood in the framework of planet formation models invoking collisional growth of planetesimals and planetary embryos.

Our planet formation model is based on core formation via pebble and gas accretion, planet migration, and disk evolution (Bitsch et al. 2015b). Planetary cores grow through the accretion of pebbles (Lambrechts \& Johansen 2014) until they reach pebble isolation mass, which is where the planet carves a partial gap in the gas disk and pebble accretion stops (Lambrechts et al. 2014). The planet then starts to accrete a gaseous envelope until $M_{\text {env }} \sim M_{\text {core }}$, which is when runaway gas accretion sets in. At the same time, the planet migrates through the disk, where we take the fully unsaturated torques into account (Paardekooper et al. 2011). The structure of the protoplanetary disk, the accretion rates for pebble accretion, and the planetary migration rates depend strongly on the metallicity (Paardekooper et al. 2011; Lambrechts \& Johansen 2014; Bitsch et al. 2015a). Here, we use a simplified disk model consisting of two power laws, one for the viscously dominated and one for the irradiation dominated regime (Ida et al. 2016). This allows us additionally to probe the dependency on the disk's viscosity. These different parameters (metallicity and viscosity) strongly influence the individual growth tracks (evolution of planetary mass as a function of semimajor axis) of the individual planets. We therefore investigate planet formation as a function of metallicity and viscosity (Figure 3$)$. For solar metallicity $([\mathrm{Fe} / \mathrm{H}]=0)$ we use a pebble-to-gas ratio of $Z_{\text {peb }}=1.35 \%$.

In each metallicity bin $(\Delta[\mathrm{Fe} / \mathrm{H}]=0.01 \mathrm{dex})$, we simulate the growth tracks of 100,000 planets that have linearly randomly spaced starting locations in the interval of 0.1-50 au. The initial planetary mass is set to the pebble transition mass, where pebble accretion starts to be efficient from the whole Hill radius of the planet (Ida et al. 2016). We assume that the disk is already $1.5 \mathrm{Myr}$ old and has a total lifetime of $3 \mathrm{Myr}$, which is well within the limits of the measurements of lifetimes of protoplanetary disks (Mamajek 2009). In principle, shorter/longer disk lifetimes can change the giant planet formation frequency (Ndugu et al. 2018), but for clarity we investigate here only the influence of a change in metallicity on the giant planet formation efficiency. This allows us to investigate the influence of metallicity on planet formation. In Figure 3, we show the fraction of giant planets $\left(M_{p}>100 M_{\oplus}\right)$ compared to the total number of planetary seeds in each metallicity bin as a function of their final orbital distance and the metallicity. Clearly, decreasing viscosity and increasing metallicity enhances planetary growth. In fact, for the highest metallicity investigated $([\mathrm{Fe} / \mathrm{H}]=0.4)$ nearly $90 \%$ of all planetary seeds grow to become gas giants. For the $\alpha$ viscosity parameters of 0.001 and 0.002 , it seems that no giant planets would form outside of 10-15 au at high metallicities. In order to form giant planets that end up at orbital positions larger than 10-15 au, the planetary seed would need to form outside of $50 \mathrm{au}$, which is larger than the interval we used for our simulations. We assume that the protoplanetary disk photoevaporates after $3 \mathrm{Myr}$. We do not take into account the possibility that disks around low-metallicity stars could have a 


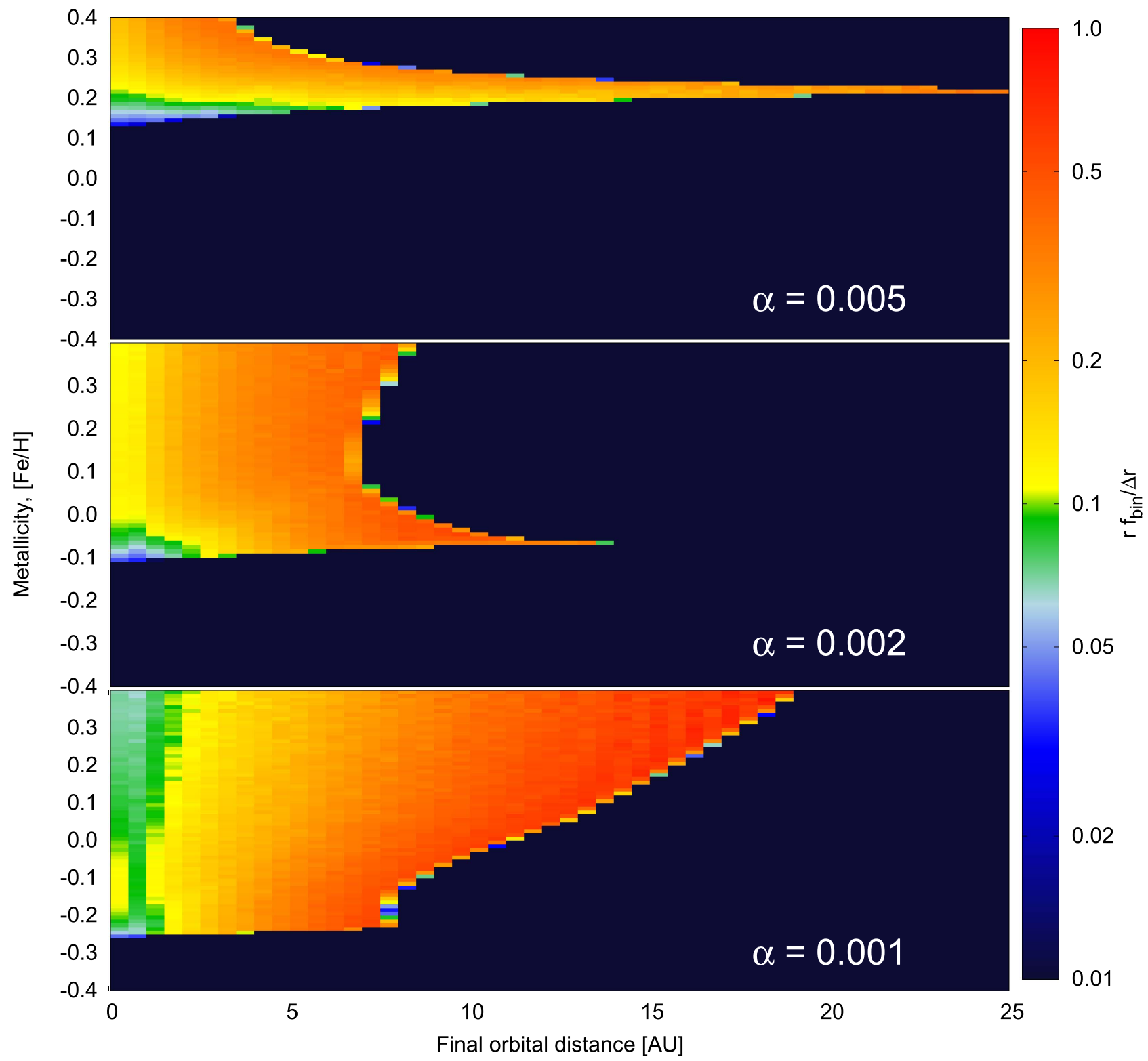

Figure 3. Fraction of planetary seeds that become gas giant planets $\left(M_{p}>100 M_{\oplus}\right)$ in planetary formation simulations, divided by the bin size and multiplied by the final orbital position to obtain the fraction per logarithmic orbital distance. This fraction is plotted as a function of the final planetary position after migration and metallicity. The panels show results for three values of the dimensionless protoplanetary disk viscosity $\alpha$ (top: $\alpha=0.005$, middle: $\alpha=0.002$, bottom: $\alpha=0.001$ ). Each metallicity line contains 100,000 planetary seeds with random starting positions distributed in the range of $0.1-50$ au. The gas giant fraction increases with increasing metallicity of the disk and with decreasing viscosity. At $\alpha=0.002$, approximately $20 \%-25 \%$ of all of the seeds form Jupiter analogs at solar metallicity $([\mathrm{Fe} / \mathrm{H}]=0)$, but the gas giant fraction rises sharply to above $50 \%$ at slightly super-solar values of the metallicity. Hence the formation of systems containing multiple cold gas giants is much more likely at elevated metallicities. Mutual scattering between these gas giants leads to ejections from the system and increases the eccentricity of the surviving planets; this can even lead to the formation of hot-Jupiter planets by tidal circularization at perihelion. This explains the trend for host stars of eccentric cool Jupiters and hot Jupiters to have elevated metallicities. True Jupiter analogs, on the other hand, emerge in systems that form only a single gas giant, a more probable outcome at solar and subsolar metallicity.

shorter lifetime to X-ray photoevaporation (Ercolano \& Clarke 2010), but adding such a dependency would simply work to amplify our key result that high-metallicity stars are more likely to form multiple giant planets.

Figure 3 shows the fraction of planetary seeds that become gas giants $\left(M_{p}>100 M_{\oplus}\right)$ formed in planetary formation simulations including pebble accretion, gas accretion, and planetary migration. The growth to giant planets is enhanced for lower viscosities, because of (i) the higher surface density for disks with constant accretion rates and (ii) because of the lower pebble scale height, which allows a faster accretion rate.
The gas giant fraction is shown as a function of the final orbital distance of the planet, the metallicity, and the $\alpha$-viscosity parameter of the disk (varied in the three panels). Decreasing the viscosity allows for a more efficient growth of the planetary seeds, because the gas column density must be higher in low viscosity disks, in order to drive the observed mass accretion rate onto the star. Increasing the disc metallicity shortens the core accretion timescale via pebble accretion, because the protoplanetary disk has more available pebbles to accrete (Bai \& Stone 2010). Although planetary seeds in our simulations start at random locations between 0.1 and $50 \mathrm{au}$, the final 
orbital distances of the gas giant planets are concentrated in the inner regions of the protoplanetary disk. This crowding of giant planets in the inner region increases with metallicity, as a larger fraction of the seeds grow to become gas giants. Therefore, high-metallicity systems are expected to contain multiple gas giants that can perturb each other gravitationally. These perturbations can lead to enhanced planet-planet scattering rates that will either eject one of the planets or leave it on an eccentric orbit and transfer the other planet to a much shorter orbital period (Lega et al. 2013), which has also been suggested by studies searching for outer gas giant companions to known exoplanets (Bryan et al. 2016). Increased metallicity can also result in an enhancement of the planetesimal formation rate (Bai \& Stone 2010). These leftover planetesimals can destabilize the giant planet configuration over longer timescales (Morbidelli et al. 2005) and further increase the eccentricity distribution of the planetary systems.

The enhanced planet-planet scattering observed in the simulations for higher metallicity systems due to the crowding of giant planets in the inner disk regions is in keeping with the observation that Jupiter analogs are found, on average, around stars of lower metallicity relative to the hot-Jupiter or eccentric cool-Jupiter exoplanets. Eccentric cool Jupiters likely experienced dynamical encounters with other gas giants, resulting in the scattering of one of the planets. Thus, only a fraction of the gas giant planets that originally formed around high-metallicity stars remain Jupiter analogs. These observations suggest that both inward migration and planet-planet scatterings, resulting in hot Jupiters and eccentric cool-Jupiter exoplanets respectively, are more common in metal-rich systems. Therefore, the initial metal content of protoplanetary disks appears to regulate the structure and architecture of planetary systems.

Another possible interpretation is that the metallicity trends could be an imprint of disk dispersal, where disk lifetimes are shorter at lower metallicities (Yasui et al. 2009; Ercolano \& Clarke 2010). The short lifetime could, on average, yield fewer multiple giant planets, and these single giant planets could migrate inward and their final location would be set by insideout photoevaporation which produces an increase of single giant planets just beyond the critical radius, 1 au (Alexander \& Pascucci 2012), corresponding to the cool circular orbit Jupiters in this analysis. The timescale for dispersing the highmetallicity disks is longer and there is therefore more time to form multiple large planets whose final locations (and eccentricities) depend on the interaction with other planets, both while migrating in the disk as well as post disk dispersal.

\section{Additional Tests and Discussion}

In order to test whether excluding the two stars with lower surface gravity, $\log g<4.0$, affected our results, we lowered our surface gravity threshold to 3.8, which resulted in HD 114613 and HD 11964 being included in the analysis. The K-S test described in Section 3 decreased slightly in significance but remained statistically significant. Subsequently, we used stellar parameters from SWEET-Cat (Santos et al. 2013) in order to include HD 154857 and HIP 57274 in our analysis. In this case, the significance of the results in the main paper increased slightly.

The Jupiter analogs and cool eccentric Jupiter planet discoveries originate from a number of RV survey programs designed to discover and measure the mass of exoplanets using precise radial velocity measurements. All of the surveys target solar-type stars that are bright and in proximity to our Solar
System, and some of the surveys, like the HARPS and CORALIE surveys, are volume-limited surveys, thus targeting all solar-type stars within a given distance. For example, the HARPS volume-limited sample (Naef et al. 2010) contains 850 F8 to M0 dwarfs within $57.5 \mathrm{pc}$. The sample of hot-Jupiter planets in this paper were discovered using the transit method and subsequently followed up with radial velocities to measure their masses. Both the RV surveys and the transit surveys suffer from inherit detection biases: larger and more massive planets in short orbital periods are more easily detected. Furthermore, there are human biases, such as prioritizing targets that exhibit variability early on because they might yield publishable results. However, the vast majority of the surveys (both RV and transit) have no significant inherent bias toward metallicity, since the samples from the majority of the surveys were selected as volume-limited samples or targeted bright solar-like stars.

Detection biases affect occurrence rate calculations and therefore, completeness studies must be undertaken to account for these effects in order to draw robust conclusions. However, this study pertains to the host-star metallicities of exoplanets belonging to different categories of planets (Jupiter analogs, eccentric cool Jupiters, and hot Jupiters) and does not address occurrence rate questions. When surveys are biased toward discovering a particular type of planet (like hot Jupiters), the detected number of planets of this particular type of planets is affected (and hence the occurrence rate, if the bias is not dealt with), but the average metallicity of the host stars of a particular type of planet (e.g., hot Jupiters) are not affected by this bias, unless there is a very strong inherent bias toward observing only a particular host-star metallicity, which is not the case.

The surveyed stars in the Kepler Mission were not selected based on host-star metallicity and neither were the surveyed stars in the ground-based transit surveys nor the RV surveys. It is also important to note that even if there was a bias toward, for example, more readily detecting planets around metal-rich stars for some reason, the results in this paper would be even more significant, since it would disfavor the detection of Jupiter analogs, which form around stars with lower average metallicities.

Furthermore, we note that the hot-Jupiter sample in this survey, which is based on planets detected using the transit method, have an average metallicity nearly identical to the hot Jupiters in the Fischer \& Valenti (2005) paper discovered using the radial velocity method (average metallicity of hot Jupiters from Fischer \& Valenti (2005) $[\mathrm{m} / \mathrm{H}]=+0.23 \pm 0.03$ and average metallicity of hot Jupiters in this paper $[\mathrm{m} / \mathrm{H}]=$ $+0.25 \pm 0.03)$. As such, biases inherit to the detection methods should not affect the result and conclusions in this paper, since we are examining the average metallicities of the host stars of planets of different types.

To test if large uncertainties on the eccentricities affect our results, we imposed a maximum eccentricity uncertainty of 0.2 , which resulted in the removal of two systems. This resulted in a change in the confidence level of the $\mathrm{K}-\mathrm{S}$ test from $99.996 \%$ $\left(4.1 \sigma, p\right.$-value $\left.=4.26 \times 10^{-5}\right)$ to $99.97 \%(3.7 \sigma, p$-value $=$ $\left.2.57 \times 10^{-4}\right)$.

In order to ensure the robustness of the results, we performed a bootstrap with a replacement analysis. We randomly drew new samples from the original samples (of the same size as the original samples) and allowed planets to be drawn more than once (with replacement) and repeated the test $10^{6}$ times. We 


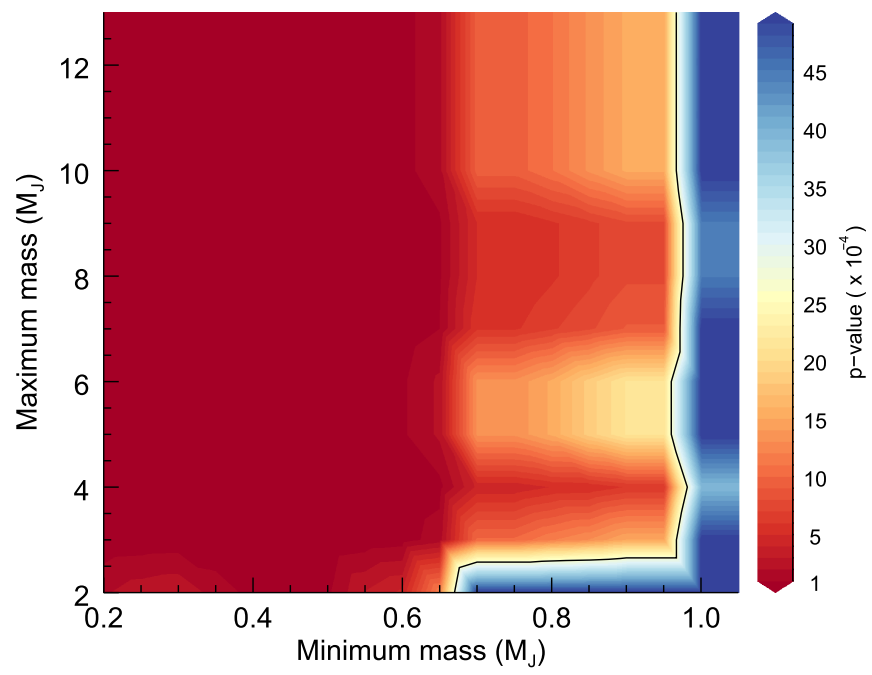

Figure 4. We performed $\mathrm{K}-\mathrm{S}$ tests on the Jupiter analogs and the hot Jupiters, testing various mass ranges. We computed the $p$-value from the $\mathrm{K}-\mathrm{S}$ test in a grid varying the minimum mass from $0.2 M_{\mathrm{J}}$ to $1.1 M_{\mathrm{J}}$ in $0.05 M_{\mathrm{J}}$ steps and the maximum mass from $2 M_{\mathrm{J}}$ to $13 M_{\mathrm{J}}$ in $1 M_{\mathrm{J}}$ steps. The resulting $p$-values are shown as color coded contours with red having a high confidence level and blue a low confidence level. The red and orange colors encapsulated by the solid black line represent $p$-values corresponding to a confidence level over $3 \sigma$.

then performed the same $\mathrm{K}-\mathrm{S}$ test for each of the draws asking with what confidence the null hypothesis that the newly drawn sample of stars orbited by Jupiter analogs and the sample of stars orbited by hot Jupiters were drawn from the same parent population could be rejected. We found that the samples were not drawn from the same parent population with $3.98_{-0.67}^{+0.60} \sigma$ confidence, in line with the conclusions in Section 3.

To further test the robustness of our results to different mass definitions of Jupiter analogs, we expanded our mass range to include planets with masses between $0.2 M_{\mathrm{J}}<M_{p} \sin i<$ $13 M_{\mathrm{J}}$. Using exoplanet.org, we found 2 planets with masses between $0.2 M_{\mathrm{J}}$ and $0.3 M_{\mathrm{J}}$ and 20 planets with masses between $3.0 M_{\mathrm{J}}$ and $13.0 M_{\mathrm{J}}$. To measure the host-star metallicities of these stars, we gathered 9 new observations from TRES and 736 public archival spectra from HARPS, which yielded reliable host-star metallicities for 17 of the 22 planets. Using the full mass range $\left(0.2 M_{\mathrm{J}}<M_{p} \sin i<13.0 M_{\mathrm{J}}\right)$, we find average metallicities of the Jupiter analogs (26 planets), the hot Jupiters (32 planets), and the cool eccentric Jupiters (28 planets) of $[\mathrm{m} / \mathrm{H}]=-0.04 \pm 0.05,[\mathrm{~m} / \mathrm{H}]=+0.24 \pm 0.03$, and $[\mathrm{m} / \mathrm{H}]=+0.17 \pm 0.03$, respectively. The two-sample $\mathrm{K}-\mathrm{S}$ test shows that the null hypothesis that the sample of stars orbited by Jupiter analogs and the sample of stars orbited by hot Jupiters are drawn from the same parent population can be rejected with $99.996 \%$ confidence $(4.1 \sigma, p$-value $=3.92 \times$ $10^{-5}$ ). Furthermore, a two-sample $\mathrm{K}-\mathrm{S}$ test shows that the null hypothesis that the sample of stars orbited by Jupiter analogs and the sample of stars orbited by cool eccentric Jupiters are drawn from the same parent population can be rejected with $99.90 \%$ confidence $\left(3.3 \sigma, p\right.$-value $\left.=9.74 \times 10^{-4}\right)$.

We then performed $\mathrm{K}-\mathrm{S}$ tests on the Jupiter analogs and the hot Jupiters, testing various mass range combinations. We computed the $p$-value from the $\mathrm{K}-\mathrm{S}$ test in a grid varying the minimum mass from $0.2 M_{\mathrm{J}}$ to $1.1 M_{\mathrm{J}}$ in $0.05 M_{\mathrm{J}}$ steps and the maximum mass from $2 M_{\mathrm{J}}$ to $13 M_{\mathrm{J}}$ in $1 M_{\mathrm{J}}$ steps. The resulting $p$-values can be seen in Figure 4, where the red and orange colors encapsulated by the solid black line represent $p$-values corresponding to a confidence level over $3 \sigma$.

To explore whether a two-component model with two averages and with a dividing eccentricity as a free parameter is a better fit to the data than a simple average or a linear model, we fitted the data using three different models using a Markov Chain Monte Carlo (MCMC) approach. We tested three models: a simple average for the entire sample, a twocomponent model with two averages and with the dividing eccentricity as a free parameter, and lastly, a linear model. We derived the maximum likelihood from the posteriors and calculated the Bayesian Information Criterion (BIC) for each model.

We found that the two-component model represented the data significantly better than the model with a simple average $(\triangle \mathrm{BIC}=120)$. The linear model was also significantly preferred over the model with a simple average $(\triangle \mathrm{BIC}=$ 106). The two-component model was thus statistically significantly favored over both of the other models. Furthermore, in order to account for the uncertainties in the eccentricities, we created 5000 new sets of data by perturbing the eccentricities within their uncertainties. For each of the 5000 sets of data, we carried out the test described above and determined the BIC values from the posteriors of the distributions. We found similar results as above: the twocomponent model was significantly favored over the model with a simple average $\left(\Delta \mathrm{BIC}=95.3_{-30.6}^{+23.8}\right)$ and the linear model was similarly favored over the simple average $(\triangle \mathrm{BIC}=$ $\left.80.1_{-31.3}^{+25.8}\right)$. And finally, as before, the two-component was on average also significantly preferred over the linear model, although with large uncertainties $\left(\triangle \mathrm{BIC}=15.9_{-43.8}^{+35.1}\right)$. These results, along with the results from the $\mathrm{K}-\mathrm{S}$ test, and the statistically significant difference in the average metallicities when considering the standard error of the mean of the two samples, shows that the results are indeed robust.

In this work, we require that the Jupiter analog planets receive less than a quarter of the insolation of the Earth $\left(S_{\text {eff }}<0.25 S_{\oplus}\right)$. To investigate the influence of this requirement on our results, we performed tests where we included planets receiving a larger amount of insolation using literature values originating from SWEET-Cat (Santos et al. 2013), which is a collection of stellar parameters for planet-hosting stars with the goal of providing a homogeneous table of stellar parameters when possible. In Figure 5, we show the metallicities of star-hosting planets with masses between $0.3 M_{\mathrm{J}}<M_{p}<3.0 M_{\mathrm{J}}$ as a function of the insolation they receive. The average metallicities of the stars in receiving an insolation of (cool) $0.01 S_{\oplus}<S_{\text {eff }}<0.25 S_{\oplus}$, (warm) $0.25 S_{\oplus}<$ $S_{\text {eff }}<10 S_{\oplus}$, and (hot) $10 S_{\oplus}<S_{\text {eff }}<10000 S_{\oplus}$ is (cool) $0.00 \pm 0.05( \pm 0.22)$, (warm) $+0.08 \pm 0.03( \pm 0.24)$, and (hot) $+0.15 \pm 0.02( \pm 0.15)$, where the uncertainty is given as the standard error of the mean and the standard deviation is in parentheses. The cool non-eccentric Jupiter analogs and the hot Jupiters show the same pattern as seen in Section 3, although the hot Jupiters are slightly less metal-rich. The warm non-eccentric Jupiters appear to have an average metallicity intermediate to the cool and hot Jupiters. We note that when using a period or semimajor axis as a proxy rather than insolation, the hot Jupiters orbit more metal-rich stars, while the differences between the medium and long period planets (or planets with a medium or large semimajor axis) are diminished, indicating the importance of using insolation to investigate the metallicity dependence. As 


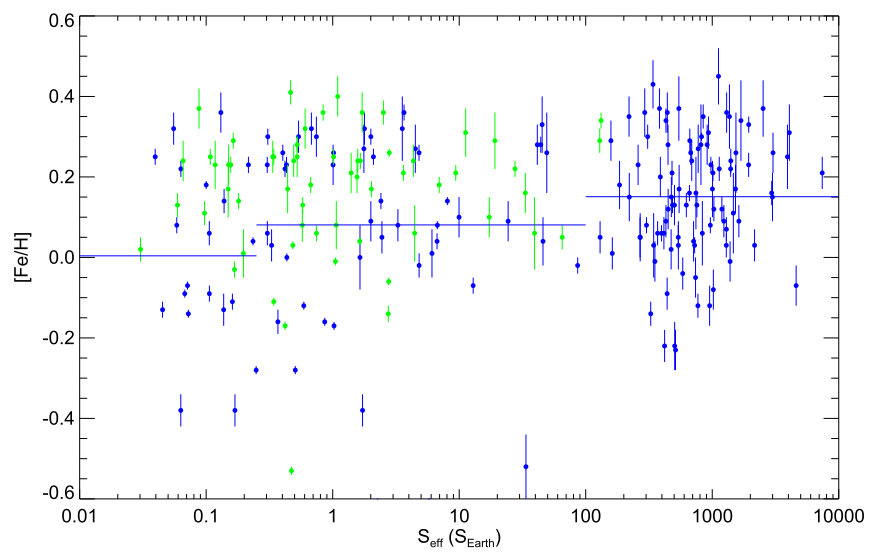

Figure 5. Metallicity of the host stars of planets with masses between $0.3 M_{\mathrm{J}}<M_{p}<3.0 M_{\mathrm{J}}$ from exoplanets.org as a function of the insolation received in units of Earth insolation. The metallicities are from the SWEET-Cat catalog using only stars that were flagged as having been homogeneously analyzed using the same method. Blue points signify stars with eccentricities of $e>0.25$ and green points are stars orbited by planets with $e>0.25$. The horizontal blue lines indicate the average metallicity of stars with planets with low eccentricity receiving insolation of $0.01 S_{\oplus}<S_{\text {eff }}<0.25 S_{\oplus}$, $0.25 S_{\oplus}<S_{\text {eff }}<10 S_{\oplus}$, and $10 S_{\oplus}<S_{\text {eff }}<10,000 S_{\oplus}$. The intermediate warm Jupiters have an average metallicity which is in between the Jupiter analogs and the hot-Jupiter type planets.

such, these results seem compatible with recent results from the LAMOST survey, where Mulders et al. (2016) find that the average metallicities of stars hosting large planets (larger than Neptune) appear to be consistently metal-rich out to a period of a few hundred days (see their Figure 8, bottom panel). However, the number of targets is small, and although not statistically significant, it appears that the close-in hot Jupiters orbit more metal-rich host stars with a slight decrease in metallicity at longer orbital periods, which is consistent with our findings when examining planets from exoplanets.org and SWEET-Cat.

We note that the average orbital period of the Jupiter analogs is 2348 days whereas Jupiter has a longer orbital period of 4332 days. We also caution that the samples of Jupiter analogs and eccentric cool Jupiter in this analysis are small since such planets are difficult to detect with current techniques.

The signal-to-noise ratio $(\mathrm{S} / \mathrm{N})$ of all of the spectra used is adequate to derive precise stellar parameters using SPC. As such, the uncertainties in the stellar parameters reflect the systematics in the stellar models and not the photon noise. Thick disk stars have a wide variation in their metallicities, but their metallicity distribution peaks at $[\mathrm{Fe} / \mathrm{H}]-0.7$. The stars in our sample are thus consistent with being thin-disk stars. As such, we conclude that neither $\mathrm{S} / \mathrm{N}$ ratio nor galactic chemical evolution could be the root cause of the average metallicity difference we observe.

\section{Conclusion}

Although a number of factors and initial conditions influence the outcome of planet formation, metallicity appears to be one of the key parameters determining which type of planets are formed and their final configuration. In this paper, we show, using homogeneously derived spectroscopic metallicities, that stars hosting Jupiter analogs have an average metallicity close to solar, in contrast to their hot-Jupiter and eccentric cool-Jupiter counterparts, which orbit stars with super-solar metallicities. And warm Jupiters seem to orbit stars with an average metallicity intermediate to that of the hot and cool Jupiters. We also find that the eccentricities of Jupiter analogs increase with host-star metallicity, suggesting that planet-planet scatterings producing highly eccentric cool Jupiters could be more common in metalrich environments. Conversely, very metal-poor systems will lack the required amount of material to form planets at all.

This suggests the existence of an intermediate metallicity regime where the formation of terrestrial planets and of a single gas giant akin to Jupiter is more likely to occur. The data at hand indicate that, on average, Jupiter analogs (this paper) and terrestrial size planets (Buchhave et al. 2012, 2014; Mulders et al. 2016) that are not in very short-period orbits form around stars with lower metallicities that are, on average, close to solar.

A direct consequence of the formation of planetary cores (with at least 10-20 Earth masses) is their feedback onto the disk structure. The planet opens up a partial gap and generates a pressure bump outside of its orbit, which is an efficient mechanism for limiting the inward flux of pebbles (Lambrechts et al. 2014). Thus, the amount of solids available to fuel planetary growth sunward of the gap may be significantly reduced, resulting in much slower growth rates in the inner disk (Morbidelli et al. 2015). If correct, this could imply that systems analogous to the Solar System with small, rocky, and potentially habitable planets might be common around stars hosting a Jupiter analog.

M.B. acknowledges financial support from the Danish National Research Foundation (\#DNRF97) and from the European Research Council (ERC Consolidator grant agreement 616027-STARDUST2ASTEROIDS). L.A.B. acknowledges financial support from the Villum Foundation YIP Program and the Carlsberg Foundation Distinguished Associate Professor Fellowship.

\section{ORCID iDs}

Lars A. Buchhave (iD https://orcid.org/0000-0003-1605-5666 Bertram Bitsch (1) https://orcid.org/0000-0002-8868-7649 David W. Latham (iD https://orcid.org/0000-0001-9911-7388 Allyson Bieryla (i) https://orcid.org/0000-0001-6637-5401 David M. Kipping (iD https://orcid.org/0000-0002-4365-7366

\section{References}

Alexander, R. D., \& Pascucci, I. 2012, MNRAS, 422, L82

Bai, X.-N., \& Stone, J. M. 2010, ApJL, 722, L220

Baruteau, C., Crida, A., Paardekooper, S.-J., et al. 2014, in Protostars and

Planets VI, ed. H. Beuther, R. S. Klessen, C. P. Dullemond, \& T. Henning (Tucson, AZ: Univ. Arizona Press), 667

Bitsch, B., Johansen, A., Lambrechts, M., \& Morbidelli, A. 2015a, A\&A, 575, A28

Bitsch, B., Lambrechts, M., \& Johansen, A. 2015b, A\&A, 582, A112

Borucki, W. J., Koch, D., Basri, G., et al. 2010, Sci, 327, 977

Brauer, F., Dullemond, C. P., Johansen, A., et al. 2007, A\&A, 469, 1169 Bryan, M. L., Knutson, H. A., Howard, A. W., et al. 2016, ApJ, 821, 89 Buchhave, L. A., Bizzarro, M., Latham, D. W., et al. 2014, Natur, 509, 593 Buchhave, L. A., Latham, D. W., Johansen, A., et al. 2012, Natur, 486, 375 Chatterjee, S., Ford, E. B., Matsumura, S., \& Rasio, F. A. 2008, ApJ, 686, 580 Dawson, R. I., \& Murray-Clay, R. A. 2013, ApJL, 767, L24

Ercolano, B., \& Clarke, C. J. 2010, MNRAS, 402, 2735

Fischer, D. A., \& Valenti, J. 2005, ApJ, 622, 1102

Fürész, G. 2008, PhD thesis, Univ. Szeged

Huber, D., Silva Aguirre, V., Matthews, J. M., et al. 2014, ApJS, 211, 2

Ida, S., Guillot, T., \& Morbidelli, A. 2016, A\&A, 591, A72

Kooten, E. M. M. E. V., Wielandt, D., Schiller, M., et al. 2016, PNAS, 113, 2011 
Lambrechts, M., \& Johansen, A. 2012, A\&A, 544, A32

Lambrechts, M., \& Johansen, A. 2014, A\&A, 572, A107

Lambrechts, M., Johansen, A., \& Morbidelli, A. 2014, A\&A, 572, A35

Lega, E., Morbidelli, A., \& Nesvorný, D. 2013, MNRAS, 431, 3494

Levison, H. F., Kretke, K. A., \& Duncan, M. J. 2015, Natur, 524, 322

Mamajek, E. E. 2009, in AIP Conf. Ser. 1158, Exoplanets and Disks: Their Formation and Diversity, ed. T. Usuda, M. Tamura, \& M. Ishii (Melville, NY: AIP), 3

Mayor, M., Marmier, M., Lovis, C., et al. 2011, arXiv:1109.2497

Mayor, M., Pepe, F., Queloz, D., et al. 2003, Msngr, 114, 20

Morbidelli, A., Bitsch, B., Crida, A., et al. 2016, Icar, 267, 368

Morbidelli, A., Lambrechts, M., Jacobson, S., \& Bitsch, B. 2015, Icar, 258, 418

Morbidelli, A., Levison, H. F., Tsiganis, K., \& Gomes, R. 2005, Natur, 435, 462

Morbidelli, A., Lunine, J. I., O’Brien, D. P., Raymond, S. N., \& Walsh, K. J. 2012, AREPS, 40, 251

Mulders, G. D., Pascucci, I., Apai, D., Frasca, A., \& Molenda-Żakowicz, J. 2016, AJ, 152, 187

Naef, D., Mayor, M., Lo Curto, G., et al. 2010, A\&A, 523, A15
Ndugu, N., Bitsch, B., \& Jurua, E. 2018, MNRAS, 474, 886

Ormel, C. W., \& Klahr, H. H. 2010, A\&A, 520, A43

Paardekooper, S.-J., Baruteau, C., \& Meru, F. 2011, MNRAS, 416, L65

Petigura, E. A., Howard, A. W., Marcy, G. W., et al. 2017, AJ, 154, 107

Pollack, J. B., Hubickyj, O., Bodenheimer, P., et al. 1996, Icar, 124, 62

Santos, N. C., Israelian, G., \& Mayor, M. 2004, A\&A, 415, 1153

Santos, N. C., Sousa, S. G., Mortier, A., et al. 2013, A\&A, 556, A150

Shabram, M., Demory, B.-O., Cisewski, J., Ford, E. B., \& Rogers, L. 2016, ApJ, 820, 93

Sousa, S. G., Santos, N. C., Israelian, G., Mayor, M., \& Udry, S. 2011, A\&A, 533,141

Telting, J., Avila, G., Buchhave, L., et al. 2014, AN, 335, 41

Tsiganis, K., Gomes, R., Morbidelli, A., \& Levison, H. F. 2005, Natur, 435, 459

Vogt, S. S., Allen, S. L., Bigelow, B. C., et al. 1994, Proc. SPIE, 2198, 362

Wang, L., Wang, W., Wu, Y., et al. 2016, AJ, 152, 6

Yasui, C., Kobayashi, N., Tokunaga, A. T., Saito, M., \& Tokoku, C. 2009 ApJ, 705, 54 